\title{
ÍNDICES E INDICADORES \\ DEL SISTEMA ELECTORAL \\ Y DEL SISTEMA DE PARTIDOS. \\ UNA PROPUESTA INFORMÁTICA \\ PARA SU CÁLCULO ${ }^{1}$
}

Francisco A. Ocaña

Universidad de Granada ${ }^{2}$

Pablo Oñate

$\mathrm{UAM}^{3}$

\begin{abstract}
RESUMEN
En estas páginas se presenta un programa informático que estará a disposición de los usuarios en la página web del CIS a partir del próximo 1 de octubre. Con el programa INDELEC pueden calcularse los más importantes índices de desproporcionalidad de los sistemas electorales, así como los más comunes para conocer las dimensiones de los sistemas de partidos: fragmentación, número de partidos, concentración, competitividad, polarización, volatilidad, voto regional y voto dual. En este artículo, y a modo de ejemplo, se aplica el programa INDELEC a los resultados agregados de las elecciones al Congreso de los Diputados celebradas en España desde 1977. En una monografía que, con el título Análisis electoral, verá la luz en el mes de septiembre en la colección Cuadernos Metodológicos del CIS, se analiza detenidamente cada indicador, apuntando sus ventajas e inconvenientes, y se aplican a las sucesivas convocatorias de los diversos tipos

${ }^{1}$ Los autores quieren dejar constancia de su agradecimiento a Rosa M. ${ }^{a}$ Medina, sin cuya "provocación» estas páginas no hubieran sido posibles. La Subdirección General de Política Interior y Procesos Electorales, del Ministerio del Interior, nos facilitó con una amabilidad y una eficacia dignas de encomio los datos electorales que les solicitamos, por lo que también queremos manifestar nuestro agradecimiento al Subdirector General, don Félix Marín Leiva. Mariano J. Valderrama Bonnet, Ana M. Aguilera del Pino, José Ramón Montero y Edurne Zoco nos apoyaron de distintas formas en la realización de este trabajo. Por último, querríamos agradecer al Centro de Investigaciones Sociológicas (a su Presidenta, doña Pilar del Castillo, y al Director del Departamento de Investigación, don Ismael Crespo) la cálida acogida que ha dispensado a este trabajo, propiciando que vea la luz.

${ }^{2}$ Departamento de Estadística e Investigación Operativa. E-mail: focana@platon.ugr.es.

3 Departamento de Derecho Constitucional, Ciencia Política y de la Administración. E-mail: pablo.onate@uam.es
\end{abstract}


de elecciones celebradas en nuestro país desde la reinstauración de la democracia y en distinto nivel de agregación y desagregación. Dibujamos, de esta forma, un mapa con las principales características de los diversos sistemas y subsistemas (estatales y autonómicos) de partidos habidos en España desde 1977.

\section{INTRODUCCIÓN}

Los sistemas de partidos han sido y son uno de los objetos de atención preferente tanto de politólogos como de ciudadanos en general, ya que de su configuración y dinámicas de funcionamiento depende, en buena medida, la deriva de la vida política de una comunidad dada. El estudio de las principales características y de los elementos identificativos que definen a un sistema o subsistema de partidos dado y que permiten compararlo con otros, esto es, el estudio de las dimensiones del sistema de partidos, constituirá, por tanto, uno de los aspectos fundamentales para conocer la configuración y el funcionamiento del sistema político en su conjunto. Y los sistemas de partidos son, ab initio al menos, una consecuencia de la distribución de las preferencias electorales de los ciudadanos, quienes manifiestan esas preferencias en forma de votos que, en virtud del sistema electoral, se convierten en distribución de autoridad gubernamental (generalmente escaños) (Rae, 1971: 14).

Todo sistema electoral genera efectos sobre los resultados electorales y el sistema de partidos resultante (Duverger, 1972: 245), efectos que pueden analizarse de diversas formas, dependiendo de qué unidad o ámbito nos interese ${ }^{4}$. Pero, tal vez, la más extendida sea aquella que atiende a las eventuales distorsiones en la relación entre la porción de votos y de escaños que logra cada partido; esto es, la que tiene en cuenta la desproporcionalidad que el sistema electoral genera al transformar los votos en distribución de escaños. De hecho, como afirma Nohlen (1981: 102 y 145), para calificar un sistema electoral como mayoritario o proporcional hay que atender a los efectos que produce en la relación escaños/votos, esto es, a la desproporcionalidad que genera.

El estudio de la distorsión de la proporcionalidad que un sistema electoral genera, así como las dimensiones del sistema de partidos, resulta, por tanto, imprescindible para poder describir adecuadamente las pautas de funciona-

${ }^{4}$ Pueden medirse los efectos del sistema electoral sobre la estabilidad gubernamental, la constitución de mayorías parlamentarias, las relaciones entre partidos — consecución de coaliciones de gobierno-, la posibilidad de que las minorías estén representadas en el Parlamento, las dinámicas internas de funcionamiento de los partidos y, por supuesto, el formato y dinámicas de funcionamiento del sistema de partidos. En términos generales, si nos atenemos al sistema de partidos, Duverger (1972: 252) distinguió entre efectos mecánicos y efectos psicológicos (entre las élites partidistas y entre los electores). Pero todos ellos, en definitiva, tienen en cuenta la desproporcionalidad que el sistema electoral arroja al transformar la distribución del voto en distribución de escaños. Acerca de los variados aspectos del sistema político sobre los que puede incidir el sistema electoral, ver, a modo de síntesis, Vallès y Bosch (1997: 118 ss.). 
miento de un sistema político dado. A ello se han dedicado en las últimas décadas un buen número de especialistas que han propuesto diversos procedimientos, vías y mecanismos para medir esas cuestiones. Prácticamente todos ellos consisten en la búsqueda de los valores de determinados índices, para lo que se deben aplicar determinadas fórmulas matemáticas a los resultados electorales. Su cálculo suele ser, por tanto, una tarea tediosa, lenta y propicia a incursión en errores.

El objeto del presente trabajo es el de presentar una herramienta informática (en la forma de programa computacional), que el lector podrá encontrar a su disposición a partir del 1 de octubre en la página web del CIS (http://www.cis.es), que le permitirá calcular de forma rápida y fácil los indicadores e índices más importantes para conocer la desproporcionalidad del sistema electoral, así como las dimensiones del sistema de partidos.

Se ha discutido bastante acerca de cuál deba ser el catálogo preciso de las dimensiones que mejor definen a un sistema de partidos y de los indicadores más adecuados para conocerlas, sin que se haya alcanzado un consenso unánime al respecto ${ }^{5}$. Sin embargo, se acepta de forma generalizada que tendremos una aproximación bastante completa al mismo si conocemos los datos relativos a la fragmentación, la competitividad, la polarización y la volatilidad que se registra en su seno. El mismo acuerdo se ha alcanzado acerca de cuáles han de ser los principales indicadores para medir sus valores.

A continuación ofrecemos una primera presentación de la aplicación del programa computacional para el cálculo de los índices relativos a la desproporcionalidad del sistema electoral, así como a las dimensiones del voto: INDELEC. En él se encuentran diversos módulos que permiten, además, la posibilidad de calcular otras versiones o modalidades de esos índices («versiones corregidas»), así como algunas propuestas alternativas ${ }^{6}$. El programa y la monografía en la que se estudian detenidamente tanto los diversos índices como su aplicación a todas las elecciones habidas en España se darán a conocer en el IV Congreso Nacional que la Asociación Española de Ciencia Política y de la Administración celebrará a finales del mes de septiembre en Granada. En estas páginas deberemos limitarnos a describir brevemente cada una de esas dimensiones

5 Pueden verse al respecto G. Bingham Powell, Contemporary democracies. Participation, stability and violence, Harvard University Press, Cambridge, Mass., 1982, pp. 75 ss.; y J. E. LANE y S. O. Ersson, Politics and society in Western Europe, Sage, Londres, 1987, pp. 155 ss.

${ }^{6}$ Como hemos dicho, ese programa estará a disposición de los usuarios en la página web del CIS a partir del 1 de octubre. Está ya en prensa la edición de una monografía de la Colección Cuadernos Metodológicos del CIS en la que, bajo el título Análisis electoral, analizamos todas estas cuestiones de forma mucho más detenida, explicando y discutiendo cada uno de los índices y sus diversas versiones, apuntando sus virtudes y defectos, así como su adecuación a un tipo de sistema electoral o a otro, o a un tipo de sistema de partidos o a otro. También se aplican y discuten en ella distintas alternativas a los índices que aquí se presentan. En la segunda parte de esa monografía aplicamos los distintos indicadores a los resultados de todos los comicios celebrados en España desde 1977, generales, autonómicos, europeos y municipales, analizando y comparando sus valores en distintos niveles de agregación y de desagregación. 
y los indicadores más importantes que se han propuesto para su medición. Para esta presentación hemos aplicado el programa mencionado a los resultados electorales para el Congreso de los Diputados de las siete convocatorias generales celebradas hasta la fecha: 1977, 1979, 1982, 1986, 1989, 1993 y 1996, poniendo someramente de manifiesto los niveles que en esos procesos electorales alcanzó la desproporcionalidad del sistema electoral, así como las principales características de los sistemas de partidos que se han sucedido en el conjunto del territorio estatal desde 1977 hasta la fecha.

Los índices e indicadores que el programa INDELEC permite calcular son, por tanto, los siguientes:

En cuanto a la desproporcionalidad:

- Índice de desproporcionalidad de Rae.

- Índice de desproporcionalidad de Loosemore y Hanby.

- Índice de desproporcionalidad Saint Lagüe.

- Índice de desproporcionalidad de cuadrados mínimos de Gallagher.

- Índice de desproporcionalidad de cuadrados mínimos corregido por Lijphart.

- Índice de la máxima desviación de Lijphart.

- Índice de sesgo en la distribución de escaños propuesto por Cox y Shugart.

- Indice de sesgo de Cox y Shugart corregido.

- Índice de sesgo robusto (en distintas versiones).

En cuanto a las dimensiones del voto o del sistema de partidos:

- Índices de fragmentación electoral y parlamentaria de Rae.

- Índices del número efectivo de partidos, electorales y parlamentarios. sugeridos por Laakso y Taagepera y Taagepera y Shugart.

- Índices de hiperfraccionamiento, electoral y parlamentario, de Kesselman y Wildgen.

- Índices del número de partidos, electorales y parlamentarios, de Molinar.

- Índices de concentración electoral y parlamentaria.

- Índices de competitividad electoral y parlamentaria.

- Índice de polarización de Sartori.

- Índices de polarización ponderada, electoral y parlamentaria.

- Índices de volatilidad total, entre bloques e intrabloques, propuestos por Pedersen y Bartolini y Mair (en sus versiones electorales y parlamentarias).

- Índice de voto regionalista.

- Índice de voto regionalista diferenciado.

- Índice de voto regional diferenciado de Lee.

- Índice de voto dual de Arian y Weiss. 
La forma de trabajar con los distintos módulos del programa es similar: los resultados electorales que servirán de base para los cálculos deben ser suministrados al programa almacenados en un fichero de texto ${ }^{7}$ al que denominaremos fichero input. Una vez realizados los cálculos, los valores de los índices no son mostrados directamente en la pantalla del ordenador, sino que son almacenados en otro fichero de texto, fichero output, que podrá manejarse con cualquier programa procesador de textos. El programa que proponemos es susceptible de ser usado, de esta forma, con una gran variedad de procesadores de texto, lo que redundará en beneficio del usuario, que podrá elaborar un informe con el mismo procesador de texto utilizado para expresar los resultados de los cálculos de los índices, visualizar fácilmente los resultados, confeccionar tablas o cuadros con los mismos, etc.

\section{LA DESPROPORCIONALIDAD DEL SISTEMA ELECTORAL. ÍNDICES PARA MEDIRLA}

Todo sistema electoral genera un cierto grado de desproporcionalidad al ser aplicado a unos resultados electorales dados, entendiendo por ésta la diferencia entre el porcentaje de escaños que un partido recibe respecto del porcentaje de voto que logró en las elecciones (Lijphart, 1994: 57). Todo sistema electoral funciona como un filtro que criba o tamiza las manifestaciones que los ciudadanos han llevado a cabo en las urnas, al distribuir posteriormente los escaños entre los partidos contendientes.

Pero la constatación de ese aspecto de la realidad electoral no se ha visto acompañada de un consenso a la hora de señalar el instrumento más adecuado para medirlo. Como señala Lijphart (1994: 57), la cuestión de cuál es el mejor mecanismo para medir la desproporcionalidad ha sido, curiosamente, mucho más controvertida que la de la determinación de los instrumentos más adecuados para medir las características fundamentales de los sistemas de partidos. En realidad, lo que ocurre es que, como han señalado diversos estudiosos (Gallagher, 1991: passim; Cox y Shugart, 1991: 348; Pennini, 1998: 3 ss.), no hay un índice que sea universalmente válido, sino que su adecuación dependerá, en gran medida, de la fórmula electoral que se esté utilizando en el sistema electo-

Consideraremos ficheros de texto a aquellos constituidos exclusivamente por datos en formato o código ASCII. La mayor parte de los procesadores de texto al uso (WordPerfect, MSWord, Edit, etc.) reconocen dicho código. Es usual que los procesadores de texto dispongan, adicionalmente, de su propio código para conferir a los documentos (ficheros) características estéticas especiales. Las autoridades electorales (éste es el caso, desde luego, del Ministerio del Interior español), o las páginas de Internet con resultados electorales, suelen suministrar los datos relativos a los resultados en este formato, por lo que resulta enormemente fácil confeccionar el fichero input, con cualquier procesador de texto. Por supuesto, en la página web del CIS de la que puede copiarse el programa para su instalación en el ordenador individual de cada usuario se proporcionan las instrucciones para hacerlo, para operar con el programa, así como para confeccionar ficheros input. 
ral que estudiemos. Es más, como dice Gallagher (1991: 38), «cada método para calcular la desproporcionalidad está inextricablemente unido a la fórmula utilizada para el reparto de los escaños. Cada fórmula de reparto de escaños genera su propio indicador de desproporcionalidad». El programa computacional que proponemos permite el cálculo de todos ellos, por lo que el usuario podrá decidir en cada caso cuál es el que mejor se ajusta al sistema que esté estudiando. En la monografía anunciada en la nota 6 analizamos detenidamente en qué consiste cada uno de los indicadores, que aquí nos tenemos que limitar prácticamente a mencionar.

Así, y siguiendo parcialmente a Lijphart (1994: 58 ss.), podemos sintetizar las ventajas e inconvenientes de algunos de estos indicadores de la siguiente forma. Todos los índices tienen en cuenta la diferencia que se registra entre el porcentaje de voto y porcentaje de escaños que recibe cada partido, pero se distinguen por la forma en la que computan esas diferencias.

Rae (1971: 84 ss.) entiende que la suma de las diferencias entre escaños y voto de cada partido debe dividirse por el número de partidos que se están teniendo en cuenta ${ }^{8}$. El indice de desproporcionalidad de Rae presenta el grave inconveniente de que acaba siendo tremendamente sensible a los pequeños partidos, cuya presencia distorsiona el valor del índice, provocando una minusvalorización de la desproporcionalidad en los sistemas multipartidistas, con varios pequeños partidos (generalmente los sistemas de representación propor(ional) $)^{9}$.

Estas dificultades se evitan, en buena medida, con el indice de desproporcionalidad de Loosemore y Hanby (1971: 467 ss.), quienes proponen dividir la suma de las diferencias absolutas entre votos y escaños de cada partido por dos, en lugar de por el número de partidos, como hacía $\mathrm{Rae}^{10}$. De esta forma, lo

${ }^{8}$ Rae propone la siguiente fórmula para calcular su índice:

$$
R=\frac{\sum_{i=1}^{n}\left|V_{i}-E_{i}\right|}{n}
$$

donde $V_{i}$ y $E_{i}$ representan, respectivamente, los votos y escaños del partido i-ésimo y $n$ el número total de partidos que tenemos en cuenta.

9 Tal sería el caso del sistema electoral español para el Congreso de los Diputados, en cuya elección casi siempre hay un alto número de pequeños partidos cuyo apoyo en las urnas es más que reducido. Rae intenta salvar este problema excluyendo del cálculo a aquellos partidos que no logren al menos el 0,5 por 100 del voto. Pero con esta decisión, por otra parte arbitraria, no resuelve el problema, al tiempo que tampoco ofrece una solución satisfactoria a la cuestión que plantean los partidos agrupados en la categoría «otros» (que no pueden ser individualizados, al aparecer agrupados en las estadísticas de resultados electorales, ni tratados conjuntamente).

${ }^{10}$ Loosemore y Hanby calculan su índice con la siguiente fórmula:

$$
L H=\frac{1}{2} \sum_{i=1}^{n}\left|E_{i}-V_{i}\right|
$$


que su índice pondrá de manifiesto será no la desproporcionalidad para cada partido, sino la que se registra para el conjunto de la elección ${ }^{11}$. El problema será ahora que la distorsión se produce en el sentido contrario: genera un sesgo del valor de la desproporcionalidad a la alta en los sistemas con varios pequeños partidos (normalmente sistemas de representación proporcional), como consecuencia del tipo de fórmula de reparto de escaños (de cociente) para la que fue concebido y que conduce a este indicador a incurrir en un buen número de «anomalías y paradojas» (Gallagher, 1991: 39) que aconsejan atender a otras propuestas.

El indice de cuadrados minimos propuesto por Gallagher (1991: 40 ss.) ofrece una buena solución a esos problemas, al reflejar en mayor medida las (escasas) grandes desviaciones entre porcentajes de voto y escaños que las (abundantes) pequeñas desviaciones, esto es, ponderando adecuadamente las desviaciones, al otorgar mucha mayor importancia a las que son de mayor calado ${ }^{12}$. Gallagher considera a los partidos que suelen aparecer en la categoría de "otros» como si fueran todos ellos un solo partido, a los efectos del cálculo. No obstante, como señala Lijphart (1994: 61), al elevarse las diferencias al cuadrado, se distorsiona la desviación de todos esos pequeños partidos agrupados, por lo que acaba siendo más adecuado despreciar esos pequeños partidos en nuestros cálculos (dando lugar a lo que denominaremos el indice de cuadrados minimos de Lijphart).

Otras propuestas basan sus cálculos en la diferencia relativa (ratio) escañosvotos de cada partido. Así, el indice Saint Lagüe tiene en cuenta la ratio escaños-votos de cada partido, así como la del conjunto del sistema ${ }^{13}$. Presenta el

Por su parte, Lijphart (1985: 10) ha propuesto dividir la suma de las desviaciones entre voto y escaños de cada partido no por dos, sino por el número efectivo de partidos de Laakso y Taagepera. Mejora las propuestas anteriores, pero sigue siendo demasiado sensible al número de partidos presentes en el sistema.

${ }^{11}$ Una modalidad que no introduce grandes diferencias con este modelo es el índice propuesto por Mackie y Rose (1982 y 1991), quienes restan de 100 el valor del índice de Loosemore y Hanby, denominando al resultado indice de proporcionalidad.

${ }_{12}$ Gallagher propone calcular la desproporcionalidad del sistema con la siguiente fórmula:

$$
G c m=\sqrt{\frac{\sum_{i=1}^{n}\left(\mathrm{~V}_{i}-E_{i}\right)^{2}}{2}}
$$

13 Acerca de este índice, ver Lijphart y Gibberd (1977: 241 y 242), así como Gallagher (1991: 41 y 42). La fórmula con la que se calcula el índice de desproporcionalidad Saint Lagüe es la siguiente:

$$
S L=\sum_{i=1, V_{i}>0}^{n} \frac{\left(E_{i}-V_{i}\right)^{2}}{V_{i}}
$$

Debe estarse avisado si se quiere aplicar este indicador a un sistema en el que se utilice una fórmula de atribución de escaños Saint Lagüe modificada (cuyo primer divisor no es 1, sino 1,4). 
inconveniente, sin embargo, de que su rango va de 0 al infinito, por lo que es menos expresivo que los anteriores índices (cuyo valor podía variar de 0 a 100).

También la fórmula de distribución de escaños D’Hondt (utilizada en España para todos los tipos de elecciones excepto para la del Senado) tiene su correspondiente índice para calcular la desproporcionalidad que el sistema genera, pese a que no tenga una lógica similar a los anteriores. La fórmula D'Hondt pretende mantener dentro de determinado nivel mínimo la sobrerrepresentación del partido más sobrerrepresentado. Por esta razón, el índice de la máxima desviación ("Lmax») debe estar expresado por el valor de la ratio escaños-votos del partido más sobrerrepresentado (que, generalmente, será el partido más votado) ${ }^{14}$.

Por otro lado, y dado que las fórmulas de representación proporcional tienden a favorecer a los partidos más votados, puede ser interesante medir este sesgo, como complemento de la información aportada por los índices de desproporcionalidad propuestos. Cox y Shugart (1991) han tratado de medirlo mediante su indice de sesgo, consistente en la pendiente (b) de la recta de regresión de los porcentajes de escaños sobre los respectivos porcentajes de voto ${ }^{15}$. Este indicador presenta el inconveniente de que es muy sensible a la presencia de pequeños partidos, que pueden provocar una "artificial» disminución de la pendiente de la recta de regresión, como Lijphart (1994: 65) ha señalado. Tratando de evitar ese inconveniente, proponemos una serie de índices de sesgo corregidos: el primero de ellos (indice de sesgo corregido) consiste en el cálculo del valor de la pendiente $(b)$ considerando sólo los datos correspondientes a los

Esta corrección pretende, precisamente, discriminar a los pequeños partidos, lo que ya supondría un serio factor de desproporcionalidad.

${ }^{14}$ Cfr. Gallagher (1991: 42). El valor de este índice de máxima desviación se calcula con la siguiente fórmula:

$$
\operatorname{Lmax}=\left\{\left|V_{i}-E_{i}\right|: i=1, \ldots, n\right\}
$$

Pese a aceptar como adecuado el índice de mínimos cuadrados de Gallagher (con las debidas correcciones), Lijphart (1994: 62) propone este índice de la máxima desviación para dar cuenta de la desproporcionalidad que arrojan los sistemas electorales, debido a su expresividad y sencillez de cálculo. No obstante, presenta el inconveniente de que sólo tiene en cuenta al partido más sobrerrepresentado, cuando pueden producirse grandes sesgos de la desproporcionalidad para los demás sin que queden registrados por este indicador.

${ }^{15}$ Esto es, se calcula a través del modelo lineal dado por la ecuación

$$
E_{i}=a+b V_{i}+\varepsilon_{i}, i=1, \ldots, n
$$

donde $\varepsilon_{i}$ es un término residual (error). El programa computacional que proponemos obtiene el valor de $b$ utilizando la fórmula clásica de ajuste lineal, tal y como estos autores propusieran inicialmente. Si el valor del índice es superior a 1,00, los partidos más grandes se habrán visto beneficiados por el sesgo; en caso de que el valor sea inferior a 1,00, el sesgo habrá jugado en favor de los pequeños partidos; mientras que si el valor fuera 1,00 no existiría sesgo, encontrándonos con una situación de perfecta proporcionalidad. 
partidos que obtienen escaños. Obviamente, este indicador deja de lado la desproporcionalidad que el sistema genera para aquellos partidos que, pese a haber conseguido cierto porcentaje de voto, no alcanzan a lograr representación parlamentaria, lo que no es algo irrelevante.

La segunda propuesta de indicador de sesgo corregido, el indice de sesgo robusto, consiste, en sus distintas versiones, en la obtención de la pendiente a través de la técnica conocida como la Linea de Tukey, cuya utilización estará recomendada cuando exista en el sistema un "pequeño" conjunto de partidos políticos que manifiesten un comportamiento distinto al resto, es decir, cuando los datos relativos a ratio votos-escaños no se ajusten bien al modelo lineal del resto de los parti$\operatorname{dos}^{16}$. Y, como decimos, también de este índice proponemos tres versiones, que se caracterizan por tenerse en cuenta para su cálculo a todos los partidos, «índice de sesgo robusto", que será demasiado sensible cuando concurran un gran número de éstos; sólo a los partidos que logran representación («indice de sesgo robusto-rep»); y sólo a los partidos que han logrado al menos el 0,5 por 100 del voto válidamente emitido ("indice de sesgo robusto-0,5»).

Como decíamos más arriba, la determinación del mejor índice para medir la desproporcionalidad vendrá dada por la fórmula con la que se repartan escaños en el sistema que nos interesa. De todas maneras, los problemas que esa relatividad presenta pueden paliarse si para los cálculos de la desproporcionalidad se tienen en cuenta datos relativos no al nivel de los distritos, sino a los del conjunto del territorio nacional o estatal, ya que en la desproporcionalidad medida en este ámbito inciden otros factores, además del tipo de fórmula proporcional que se esté usando ${ }^{17}$. Y esto haremos a continuación, para presentar los valores que asume en el caso español cada indicador.

\section{LA DESPROPORCIONALIDAD EN LA ELECCIÓN DEL CONGRESO DE LOS DIPUTADOS}

Como es sabido, la desproporcionalidad del sistema electoral vendrá dada, fundamentalmente, por la configuración de los elementos de éste, en especial por el tamaño o la magnitud de los distritos o circunscripciones electorales, siendo éste el factor decisivo (Rae, 1971: 114 ss.; Taagepera y Shugart, 1990:

${ }_{16}$ Para el cálculo del índice de sesgo robusto proponemos la estimación del valor de la pendiente $b$ mediante la técnica conocida como la Línea de Tukey. Acerca del procedimiento para la obtención de esta línea puede verse cualquier obra general de estadística descriptiva o, más en concreto, en alguna obra relativa al análisis exploratorio de datos. A ellas nos remitimos, siendo la referencia básica, no obstante, el trabajo de J. W. TUKEY, Exploratory Daya Analysis, Addison Wesley, Mass., 1977.

17 Estos otros factores son, como diversos autores han señalado (Gallagher, 1991: 43; Cox y Shugart, 1991: 350; y Lijphart, 1994: 63), la magnitud del distrito, la distribución del voto y, de menor relevancia, el malapportionment (considerablemente relevante en la elección del Congreso de los Diputados) y la existencia de barreras legales o cláusulas de exclusión. 
112). Para la elección del Congreso de los Diputados se utilizan circunscripciones de magnitud bastante reducida: su promedio es de 6,7 escaños, prácticamente el límite mínimo imprescindible para que la fórmula electoral deje de tener efectos irremediablemente mayoritarios, como Montero (1998: 39) ha señalado. La consecuencia será que en muchos de los distritos pequeños la elección registrará unos considerables sesgos mayoritarios, generándose lo que Baras y Botella (1996: 104) han calificado como «bipartidismo circunscripcional». La relación entre nivel de desproporcionalidad y tamaño de la circunscripción puede apreciarse en la tabla 1, en la que se recogen los valores resultantes de aplicar el índice de cuadrados mínimos de Lijphart en el nivel de la circunscripción, agrupando a éstas en atención a su tamaño. En los distritos en los que se eligen menos de 9 escaños (tipos I, II y III) el nivel de desproporcionalidad registrado alcanza la tasa típica de los países que utilizan un sistema electoral mayoritario.

\section{TABLA 1}

Desproporcionalidad* en España en función del tamaño de las circunscripciones (1977-1996)

\begin{tabular}{|c|c|c|c|c|c|c|}
\hline & $I$ & $I I$ & III & $I V$ & $V$ & Media \\
\hline 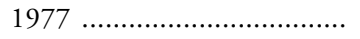 & 44,6 & 16,8 & 13,9 & 10,7 & 2,9 & 10,6 \\
\hline . & 52,8 & 16,2 & 12,1 & 10,8 & 4,6 & 11,8 \\
\hline 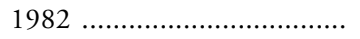 & 51,6 & 15,0 & 12,0 & 9,4 & 4,1 & 8,4 \\
\hline 1986 & 46,6 & 14,0 & 10,5 & 8,6 & 3,9 & 7,4 \\
\hline 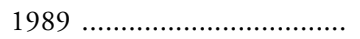 & 50,8 & 16,6 & 12,6 & 8,6 & 4,2 & 9,1 \\
\hline 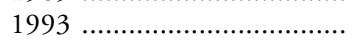 & 46,6 & 13,9 & 10,7 & 7,7 & 2,6 & 7,9 \\
\hline 1996 & 43,7 & 12,7 & 9,3 & 5,3 & 2,0 & 4,4 \\
\hline
\end{tabular}

* Índice de cuadrados mínimos de Gallagher perfilado por Lijphart. Tipos de circunscripciones: Tipo I: 1 escaño ( 2 casos en la elección de 1996). Tipo II: de 3 a 5 escaños (28 casos en la elección de 1996). Tipo III: de 6 a 8 escaños (11 casos en la elección de 1996). Tipo IV: de 9 a 30 escaños (9 casos en la elección de 1996). Tipo V: más de 30 escaños (2 casos en la elección de 1996).

Pero si se atiende a los datos agregados en el nivel estatal se observa que la consecuencia de ese pequeño tamaño de distritos es que el sistema electoral para elegir el Congreso de los Diputados arroja, efectivamente, unos altos niveles de desproporcionalidad que benefician, fundamentalmente, a los dos primeros partidos en detrimento de los que ocupan la tercera y cuarta posición. De hecho, la desproporcionalidad del sistema español está entre las más elevadas si se compara con las que se registran en los sistemas electorales del «entorno", siendo superior a la registrada en cualquier otro país que utilice un sistema de representación proporcional (sistema teóricamente utilizado en nuestro 
país), y colocándose entre los de los países que utilizan un sistema electoral mayoritario o superando incluso el de alguno de éstos (Gallagher, 1991: 46).

Sea cual sea el indicador que se utilice, la desproporcionalidad que arroja el sistema electoral que rige la elección del Congreso de los Diputados español alcanza elevadas cotas. En la tabla 2 hemos recogido los valores resultantes de aplicar distintos índices de desproporcionalidad a los resultados de las elecciones legislativas en España.

\section{TABLA 2}

Indices de desproporcionalidad para las elecciones al Congreso de los Diputados en España (1977-1996)

\begin{tabular}{|c|c|c|c|c|c|c|}
\hline Año & $S L$ & Rco & $L H$ & $\operatorname{Lmax}$ & $G m c$ & $L m c$ \\
\hline 1977 & 18,21 & 2,65 & 18,30 & 12,90 & 10,62 & 10,60 \\
\hline 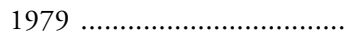 & 17,65 & 2,35 & 17,54 & 13,05 & 10,54 & 10,52 \\
\hline 1982 & 12,67 & 2,43 & 13,87 & 9,38 & 8,17 & 8,14 \\
\hline 1986 & 11,28 & 2,13 & 12,69 & 8,24 & 7,35 & 7,32 \\
\hline 1989 & 13,72 & 1,90 & 15,10 & 10,12 & 8,97 & 8,95 \\
\hline 1993 & 10,61 & 1,63 & 12,01 & 6,33 & 6,82 & 6,80 \\
\hline 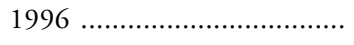 & 5,69 & 1,42 & 8,08 & 5,40 & 5,33 & 5,32 \\
\hline 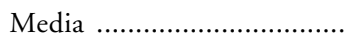 & 12,83 & 2,07 & 13,94 & 9,35 & 8,26 & 8,23 \\
\hline
\end{tabular}

SL = índice de Saint Lagüe; Rco = índice de Rae corregido; LH = índice de Loosemore y Hanby; Lmax = índice de máxima desviación de Lijphart (o índice D’Hondt); Gcm = índice de cuadrados mínimos de Gallagher; Lcm = índice de cuadrados mínimos de Lijphart.

La correlación entre los diversos índices es, en este nivel estatal de agregación, muy alta. El índice de Rae es el que menor nivel de correlación presenta, variando entre 0,832 y 0,860 , mientras que los de los demás oscilan entre 0,970 y 1,000$)^{18}$.

Estas altas tasas de desproporcionalidad obligan a recalificar el sistema electoral español, que debe ser etiquetado como sistema mayoritario atenuado, más que como de representación proporcional corregido o imperfecto ${ }^{19}$. El sis-

${ }^{18}$ Por razones de espacio, no podemos incluir aquí una matriz de correlación entre los diversos índices, por lo que remitimos al lector interesado a la monografía mencionada en la nota 6 . Los índices de sesgo diseñan un panorama similar, apareciendo beneficiados por el sesgo desproporcional en todos los casos los partidos más votados. No obstante, ninguno de los índices propuestos recogen adecuadamente el grado del sesgo a favor de esos grandes partidos, tal vez debido a la agregación de los datos en el nivel estatal. Tampoco la correlación entre los valores de los diversos indicadores es tan satisfactoria como la registrada con los anteriores índices. De todo ello nos ocupamos en la monografía mencionada.

19 Cfr., en este sentido, Montero y Vallès (1994: 8), Baras y Botella (1996), Vallès y Bosch (1997: 248 ss.) o Montero (1998: 46), entre otros. 
tema electoral español se ha manifestado, por mor de la configuración de sus distritos de reducida magnitud, como un sistema altamente desproporcional; como un potente reductor de la proporcionalidad generador de considerables efectos tanto mecánicos como psicológicos ${ }^{20}$. Debe recordarse, no obstante, que esos efectos se buscaron conscientemente cuando, al establecerse el sistema electoral, se adoptó la decisión política de primar la lógica de la estabilidad y de la gobernabilidad frente a la de la representación ${ }^{21}$.

\section{LAS DIMENSIONES DEL SISTEMA DE PARTIDOS. LOS ÍNDICES PARA MEDIRLAS}

La dimensión de la fragmentación es, sin duda, una de las más importantes ya que nos informa acerca de la medida en la que el poder político se halla disperso o concentrado. Para medirla se han propuesto distintos índices: el índice de fragmentación ${ }^{22}$; el indice de concentración ${ }^{23}$; el indice del número efectivo de partidos $^{24}$; el indice de hiperfraccionamiento ${ }^{25}$; y el indice del número de

${ }^{20} \mathrm{Ni}$ la fórmula electoral utilizada (fórmula D’Hondt) ni el umbral de representación que se aplica (3 por 100 respecto del total del voto válidamente emitido en el distrito respectivo) son los responsables de tan altas tasas de desproporcionalidad, como Vallès (1998: 79) o Botella (1998: 100) han señalado recientemente.

${ }^{21}$ Recuérdese que el sistema electoral se estableció, originariamente, por el Real Decreto-Ley 20/1977, de 18 de marzo; y, más tarde, por la Ley Orgánica 5/1985, de 19 de junio, de Régimen Electoral General (que recogió los elementos básicos de la regulación anterior). Sobre todas estas cuestiones, ver Del Castillo (1998) y Montero (1998).

22 Rae (1971: 56) calcula la fragmentación con la siguiente fórmula:

$$
F=1-\sum_{i=1}^{n} P_{i}^{2}
$$

donde $P i$ es la proporción de voto o de escaños (versión electoral o parlamentaria de la fragmentación) que consigue cada partido.

${ }^{23}$ Este índice mide el porcentaje de voto y de escaños que suman entre los dos primeros partidos.

${ }^{24}$ Propuesto por Laakso y Taagepera (1979: 3 ss.) y posteriormente perfilado por Taagepera y Shugart (1989: 77 ss.). El índice del número efectivo de partidos se calcula con la siguiente fórmula:

$$
N=\frac{1}{\sum_{i=1}^{n} P_{i}^{2}}
$$

${ }^{25}$ Fue propuesto por Kesselman (1966) y Wildgen (1971). Estos autores calculan su índice de hiperfraccionamiento con la siguiente fórmula:

$$
I=\exp \left(-\sum_{i=1, P_{i}>0}^{n} P_{i} \log \left(P_{i}\right)\right)
$$

siendo log el logaritmo neperiano. 
partidos $^{26}$. No podemos detenernos aquí en la explicación de las ventajas e inconvenientes que cada uno de estos indicadores presenta frente a los demás. Recordemos una vez más que la intención de estas páginas es presentar el programa informático que permite calcularlos, remitiendo al lector interesado a la primera parte de la monografía anunciada con anterioridad.

En la tabla 3 se han recogido los valores de la fragmentación y la concentración para las elecciones legislativas en España. Como puede observarse, se han alcanzado unos reducidos niveles de fragmentación, tanto en su versión electoral como en la parlamentaria (estos últimos más reducidos como consecuencia de los sesgos del sistema electoral). La concentración en los dos primeros partidos ha sido, correlativamente, elevada, dando lugar a mayorías suficientes que han permitido gobernar al partido ganador, aunque en algunas ocasiones haya tenido que apoyarse en pequeños partidos para alcanzar la mayoría absoluta en el Congreso de los Diputados ${ }^{27}$. Destaca, por último, la estabilidad en estos valores a lo largo de los casi veinte años que separan la primera de la última elección.

Los diversos índices que miden de distinta forma el número de partidos presentan para el caso de las elecciones legislativas unos valores coherentes con los apuntados por los de fragmentación y concentración. Como se observa en la tabla $4^{28}$, el número de partidos en España es relativamente bajo: pese a las variaciones que se han registrado (especialmente con motivo del realineamiento de 1982), el valor de uno y otro número —electoral y parlamentariosigue siendo bajo si se compara con el de los países del entorno europeo: España se sitúa entre los que registran un menor número de partidos, próxima a las posiciones de Irlanda, Alemania, Austria, Grecia y Reino Unido, que son los que más bajo índice de partidos registran (Montero, 1994: 72, y Morlino, 1995: 324 ss.).

${ }^{26}$ Para calcular el índice del número de partidos, Molinar (1991: 1383 ss.) propone la siguiente fórmula:

$$
N P=1+N^{2} \sum_{i=2}^{n} P_{i}^{2}
$$

${ }^{27}$ Esta alta concentración no ha sido incompatible con que el Congreso de los Diputados contara en casi todas las legislaturas con la presencia de un buen número de pequeños partidos de ámbito regionalista o nacionalista, sin que la estabilidad del sistema quedara, por ello, socavada. La cifra de partidos que en cada legislatura han tenido representación en el Congreso de los Diputados ha superado la decena. De esta forma, las minorías nacionalistas y regionalistas han tenido ocasión de dejar oír su voz en la Cámara, coadyuvándose así a la legitimidad del sistema democrático.

${ }^{28}$ En esta tabla 4 se puede comprobar la distinta adecuación de cada uno de los índices propuestos. Obsérvese que así como el valor del número efectivo de partidos es prácticamente igual para los años 1982 y 1996, y el de hiperfraccionamiento manifiesta un número menor en 1996 que en 1982, el del número de partidos de Molinar sí registra en el sentido adecuado las considerables diferencias que se dieron entre una y otra convocatoria. Manifiesta mejor la competitividad entre los dos primeros partidos, así como las posibilidades de formar coaliciones parlamentarias. 


\section{TABLA 3}

Fragmentación y concentración electorales y parlamentarias en España (1977-1996)

\begin{tabular}{|c|c|c|c|c|}
\hline \multirow[b]{2}{*}{ Años } & \multicolumn{2}{|c|}{ Fragmentación } & \multicolumn{2}{|c|}{ Concentración } \\
\hline & Electoral & Parlamentaria & Electoral & Parlamentaria \\
\hline 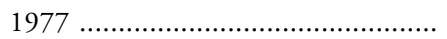 & 0,775 & 0,654 & 63,9 & 81,1 \\
\hline . & 0,767 & 0,644 & 65,5 & 82,6 \\
\hline . & 0,687 & 0,571 & 74,8 & 88,3 \\
\hline 1986 & 0,721 & 0,627 & 70,5 & 82,6 \\
\hline 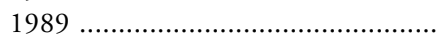 & 0,755 & 0,649 & 65,8 & 80,6 \\
\hline 1993 & 0,712 & 0,626 & 74,1 & 85,7 \\
\hline 1996 & 0,688 & 0,633 & 77,2 & 84,9 \\
\hline 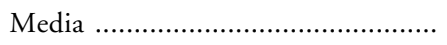 & 0,730 & 0,630 & 70,3 & 83,7 \\
\hline
\end{tabular}

\section{TABLA 4}

Número de partidos electorales y parlamentarios en España (1977-1996)

\begin{tabular}{|c|c|c|c|c|c|c|c|c|c|}
\hline & \multicolumn{3}{|c|}{ Electorales } & \multicolumn{3}{|c|}{ Parlamentarios } & \multicolumn{3}{|c|}{ Diferencia } \\
\hline & $N$ & $I$ & $N P$ & $N$ & $I$ & $N P$ & $N$ & $I$ & $N P$ \\
\hline 1977. & 4,45 & 7,45 & 3,09 & 2,89 & 3,61 & 2,01 & 1,56 & 3,84 & 1,08 \\
\hline 1979. & 4,29 & 7,23 & 3,04 & 2,81 & 3,46 & 1,99 & 1,49 & 3,77 & 1,05 \\
\hline $1982 \ldots \ldots \ldots \ldots \ldots \ldots \ldots \ldots \ldots \ldots$ & 3,20 & 5,24 & 1,81 & 2,33 & 3,06 & 1,52 & 0,87 & 2,19 & 0,29 \\
\hline 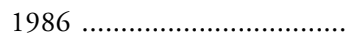 & 3,59 & 5,93 & 2,06 & 2,68 & 3,38 & 1,69 & 0,90 & 2,55 & 0,37 \\
\hline 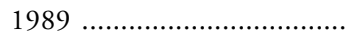 & 4,09 & 6,80 & 2,43 & 2,85 & 3,31 & 1,82 & 1,24 & 3,49 & 0,61 \\
\hline 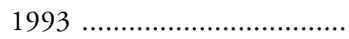 & 3,47 & 5,43 & 2,63 & 2,67 & 2,78 & 2,20 & 0,79 & 2,65 & 0,43 \\
\hline 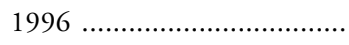 & 3,21 & 4,58 & 2,63 & 2,72 & 3,40 & 2,25 & 0,48 & 1,18 & 0,38 \\
\hline 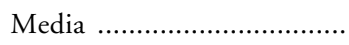 & 3,76 & 6,09 & 2,53 & 2,70 & 3,28 & 1,92 & 1,04 & 2,81 & 0,60 \\
\hline
\end{tabular}

$N=$ índice de número efectivo de partidos de Laakso y Taagepera; $I=$ índice de hiperfraccionamiento de Kesselman y Wildgen; $N P=$ índice de número de partidos de Molinar. La diferencia entre el valor del número de partidos electorales y parlamentarios duplica, en el caso de España, la media de las diferencias europeas. Esa enorme diferencia hay que buscarla, sin duda, en la alta desproporcionalidad que arroja el sistema electoral.

La competitividad manifiesta el grado de rivalidad entre los principales partidos y puede, así, ser adecuadamente medida por la proximidad de sus respectivos resultados. Los datos de la tabla 5 manifiestan las considerables variaciones que ha registrado la competitividad en España entre 1977 y 1996, que evidencian los tres períodos electorales en los que se pueden dividir las elecciones 
habidas hasta la fecha (Montero, 1997: 393 ss.): un primer período con alta competitividad, seguido de una considerable reducción con el cataclismo electoral de 1982, que inaugura el segundo período; un paulatino incremento de la competitividad en las elecciones de 1986 y 1989, hasta que en 1993 se inaugura el tercer período, en el que la competitividad aumenta hasta los niveles de la etapa de la transición; será en 1996 cuando se registren los mayores niveles de competitividad, al separar al PP del PSOE tan sólo 340.000 votos (de un censo de unos 25 millones de electores).

TABLA 5

Competitividad electoral y parlamentaria en España (1977-1996)

\begin{tabular}{|c|c|c|}
\hline Años & Electoral & Parlamentaria \\
\hline 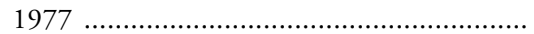 & 5,14 & 13,71 \\
\hline (1) & 4,45 & 13,43 \\
\hline 1982 & 21,86 & 27,14 \\
\hline 1986 & 18,20 & 22,57 \\
\hline 1989 & 13,90 & 19,43 \\
\hline 1993 & 4,05 & 5,14 \\
\hline 1996 & 1,17 & 4,29 \\
\hline Media & 9,82 & 15,10 \\
\hline
\end{tabular}

Recuérdese que a mayor valor del índice, menor será la competitividad.

La polarización es una de las dimensiones más relevantes del sistema de partidos, ya que constituye la variable con mayor valor explicativo a la hora de hablar de la estabilidad del sistema democrático, su eficacia, actividad y viabilidad (Sani y Sartori, 1983: 337). La polarización da cuenta de la distancia que separa en el espectro ideológico a los partidos de un sistema dado, midiéndose, habitualmente por la distancia que separa a los partidos en atención a la dimensión ideológica izquierda-derecha (Sani y Sartori, 1983: 310 ss.; Klingemann, 1995: 186 ss.; y Hazan, 1997: 15 ss.), aunque nada se opone a que se use otra que sea relevante en el sistema objeto de estudio (como, por ejemplo, la dimensión centro-periferia). Los partidos son ubicados en una escala de diez puntos (por los electores o por expertos), informando los distintos índices de polarización de la distancia que separa a los partidos en esa dimensión relevante. El indice de polarización de Sartori (PSa) da cuenta de la distancia máxima entre los dos partidos ubicados más en los extremos en cada sistema de partidos. Presenta el inconveniente de que puede distorsionar el índice al conferir igual relevancia a todos los partidos, por pequeños que sean. El indice de polarización ponderada ( $P P e$ o PPp) salva esta deficiencia, al tener en cuenta la importancia relativa de cada partido (medida en términos de votos o de esca- 
ños). Por las mencionadas restricciones de espacio a las que nos vemos sometidos, no vamos a presentar aquí resultados de la aplicación de esta dimensión al caso español, remitiéndonos nuevamente a la monografía mencionada ${ }^{29}$.

La volatilidad electoral nos informa acerca de los cambios electorales netos entre dos elecciones sucesivas que se deben a transferencias individuales del voto, poniéndose de manifiesto el grado en el que el sistema de partidos está o no cristalizado, si es estable y si la o las dimensiones en torno a las cuales ha venido articulándose siguen siendo o no relevantes (Pedersen, 1983: 31 y 32, y Bartolini y Mair, 1990: 19). Pero dentro de la volatilidad agregada debemos distinguir tres indicadores: el de la volatilidad total (VT) que se registra en el conjunto del sistema de partidos ${ }^{30}$; el de la volatilidad entre bloques de partidos (VB), agrupados en atención a una dimensión relevante (normalmente la ideológica), y el de la volatilidad intrabloques (VIB), que mide los cambios en los apoyos electorales entre los partidos agrupados en un mismo bloque ${ }^{31}$. El pro-

${ }^{29}$ El índice de polarización ponderada se calcula con la siguiente fórmula:

$$
P P=\sum_{i=1}^{n} \frac{V_{i}}{100}(x i-\bar{x})^{2}
$$

siendo $V_{i}$ el porcentaje de votos o de escaños (polarización ponderada electoral o polarizaación ponderada parlamentaria) de cada partido; $x_{i}$ la posición que ocupa el partido en la escala 1-10; y $\bar{x}$ el promedio de las posiciones de los partidos en la escala.

${ }^{30}$ Tanto Pedersen (1983: 31 y 32) como Bartolini y Mair (1990: 20 ss. y 312) utilizan la siguiente fórmula para calcular la volatilidad total:

$$
V T=\frac{1}{2} \sum_{i=1}^{n}\left|P_{i} \nabla\right|
$$

donde la variación del voto que experimenta cada partido entre dos elecciones sucesivas se representa por

$$
P_{i} \nabla=P_{i}(t+1)-P_{i}(t), \quad i=1, \ldots, n
$$

siendo $P_{i}$ la proporción de voto del partido i-ésimo y $(t)$ y $(t+1)$ dos convocatorias electorales sucesivas. El índice oscilará entre un mínimo de 0 y un máximo de 100.

${ }^{31}$ Acerca de la pertinencia de distinguir estos tipos de volatilidad para poder conocer (aún así, con cautelas) la persistencia de la relevancia de determinado cleavage en un sistema de partidos dado, ver Bartolini y Mair (1990: 36 ss.). Para la ubicación de los partidos de cada sistema occidental en uno u otro bloque puede seguirse la clasificación ofrecida por Castles y Mair (1984) y Huber e Inglehart (1995). Bartolini y Mair (1990: 22, 23 y 313) calculan la volatilidad entre bloques con la siguiente fórmula:

$$
V B=\frac{\left|P_{i} \nabla+P_{j} \nabla+\ldots+P_{k} \nabla\right|+\left|P_{x} \nabla+P_{y} \nabla+\ldots+P_{z} \nabla\right|}{2}
$$

en la que $i, j, \ldots, k$ representan partidos agrupados en un bloque, $\mathrm{y} x, y, \ldots, z$ representan partidos agrupados en el otro bloque. La volatilidad que se registra dentro de cada bloque o volatilidad intrabloques se calcula restando de la volatilidad total la volatilidad entre bloques, esto es, VIB = VT - VB. 
grama INDELEC permite calcular estos índices tanto en su versión electoral como parlamentaria.

Pues bien, unos altos niveles de volatilidad total denotarán que el sistema de partidos está experimentando serios procesos de transformación que impiden hablar de que esté cristalizado o consolidado. Así ocurriría en el caso español, en el que se han registrado unos elevados niveles de volatilidad total, si se compara con la alcanzada en otros países europeos, como se aprecia en la tabla 6 .

Pero este alto promedio de volatilidad total para el período 1977-1996 debe ser matizado con los datos relativos a los otros tipos de volatilidad. De hecho, como se puede observar en la tabla 7 , la tasa de volatilidad total ha variado considerablemente de un par de elecciones a otro: fue muy reducida, para lo que suele ser habitual tras un proceso de transición a la democracia ${ }^{32}$, en las segundas elecciones democráticas. El ajuste del sistema de partidos se produjo con la siguiente convocatoria, en la que la volatilidad alcanzó niveles desconocidos en los países europeos, y que permitieron tildar a aquellas elecciones como "cataclismo electoral». Desde entonces el nivel de volatilidad total ha tendido a reducirse, alcanzando en la actualidad niveles parejos a los que se registran en otros países europeos del entorno. Ello es indicativo de que el sistema de partidos ha ido cristalizándose a lo largo de los años, encontrándose en la actualidad bastante consolidado, por lo que no es previsible que se registren en él cambios bruscos.

\section{TABLA 6}

Volatilidad total, entre bloques e intrabloques en Europa (1968-1998)

\begin{tabular}{|c|c|c|c|c|c|c|}
\hline Pais & Años & $N .^{o}$ elecciones & $V T$ & $V B$ & $V I B$ & $\% V B / V T$ \\
\hline Alemania .................. & $1972-94$ & 6 & 6,1 & 3,6 & 2,5 & 54,8 \\
\hline Austria ...................... & $1971-96$ & 7 & 5,7 & 2,1 & 3,6 & 38,4 \\
\hline Bélgica ........................ & $1971-95$ & 8 & 7,1 & 1,2 & 5,9 & 23,9 \\
\hline Dinamarca ................ & $1971-95$ & 10 & 12,5 & 3,0 & 9,5 & 25,2 \\
\hline 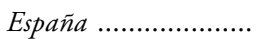 & $1977-96$ & 7 & 15,3 & 4,6 & 10,7 & 30,1 \\
\hline Finlandia ...................... & $1972-95$ & 6 & 8,9 & 2,9 & 6,0 & 32,4 \\
\hline Francia ....................... & $1968-97$ & 7 & 12,5 & 6,0 & 6,5 & 47,7 \\
\hline Grecia ...................... & $1974-96$ & 8 & 11,8 & 6,8 & 5,0 & 49,2 \\
\hline Holanda ...................... & $1971-98$ & 8 & 11,4 & 2,7 & 8,7 & 27,2 \\
\hline Irlanda ........................ & $1969-97$ & 9 & 8,0 & 2,7 & 5,3 & 36,4 \\
\hline Italia ........................... & $1968-96$ & 9 & 12,7 & 3,2 & 9,5 & 28,3 \\
\hline Noruega ..................... & $1969-97$ & 7 & 13,0 & 2,8 & 10,2 & 28,3 \\
\hline Portugal .................... & $1975-95$ & 8 & 13,9 & 5,0 & 8,9 & 37,0 \\
\hline R. Unido .................... & $1970-97$ & 7 & 6,8 & 4,3 & 2,5 & 72,8 \\
\hline Suecia ........................... & $1970-94$ & 7 & 8,0 & 3,8 & 4,2 & 43,5 \\
\hline Suiza …....................... & $1971-95$ & 6 & 5,8 & 1,3 & 4,5 & 24,0 \\
\hline Promedio ................... & $1968-98$ & 7,5 & 10,0 & 3,5 & 6,5 & 37,4 \\
\hline
\end{tabular}

32 Cfr. Gunther y Montero (1994), Morlino y Montero (1995) y Morlino (1995). 


\section{TABLA 7}

Volatilidad electoral en España (1977-1996)

\begin{tabular}{|c|c|c|c|}
\hline Años & $V T$ & $V B$ & $V I B$ \\
\hline $1979-77$.................... & 9,9 & $\begin{array}{r}1,9 \\
(19,2 \%)\end{array}$ & $\begin{array}{r}8,0 \\
(80,8 \%)\end{array}$ \\
\hline 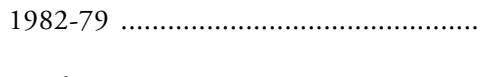 & 42,8 & $\begin{array}{r}6,3 \\
(14,7 \%)\end{array}$ & $\begin{array}{r}36,5 \\
(85,3 \%)\end{array}$ \\
\hline 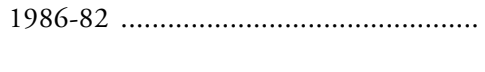 & 12,1 & $\begin{array}{r}1,7 \\
(14,1 \%)\end{array}$ & $\begin{array}{r}10,4 \\
(86,0 \%)\end{array}$ \\
\hline 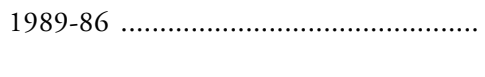 & 9,4 & $\begin{array}{r}0,5 \\
(5,1 \%)\end{array}$ & $\begin{array}{r}8,9 \\
(94,9 \%)\end{array}$ \\
\hline 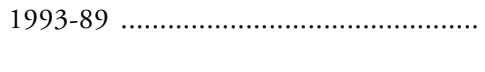 & 12,4 & $\begin{array}{r}8,6 \\
(69,0 \%)\end{array}$ & $\begin{array}{r}3,9 \\
(31,0 \%)\end{array}$ \\
\hline 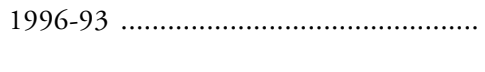 & 5,3 & $\begin{array}{r}2,8 \\
(52,2 \%)\end{array}$ & $\begin{array}{r}2,5 \\
(47,8 \%)\end{array}$ \\
\hline 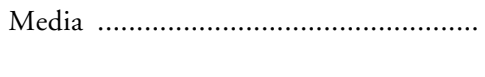 & 15,3 & $\begin{array}{r}4,6 \\
(15,6 \%)\end{array}$ & $\begin{array}{r}10,7 \\
(84,4 \%)\end{array}$ \\
\hline
\end{tabular}

$\mathrm{VT}=$ volatilidad total $\mathrm{VB}=$ volatilidad entre bloques; $\mathrm{VIB}=$ volatilidad intrabloques. La cifra entre paréntesis representa el porcentaje de la volatilidad total que supone la entre bloques o la intrabloques.

Pero, tal vez, más relevante que esta tendencia a la reducción de la tasa de volatilidad total es que, en su mayor parte, se debe a transferencias de voto entre partidos pertenecientes al mismo bloque ideológico, esto es, se trata principalmente de volatilidad intrabloques. Aunque, como Bartolini y Mair (1990: 38 ss.) han puesto de manifiesto, hay toda una serie de factores que pueden incidir en la relevancia de la barrera ideológica para un sistema de partidos dado, el hecho de que los ciudadanos que modifican su voto opten por otro partido que se encuentra en el mismo bloque ideológico que el primero implica que esa barrera sigue siendo relevante para ordenar las preferencias electorales de los ciudadanos ${ }^{33}$. Como ha concluido Montero (1997: 418), el cleavage que divide actualmente al sistema de partidos en España "parece expresarse fundamentalmente a través de una visión de la política asociada con los términos espaciales de izquierda-derecha», que, por mucho que sus contenidos sean imprecisos y variables, siguen funcionando como mecanismo para ordenar el comportamiento político de los ciudadanos españoles.

Pero también pueden estudiarse los sistemas de partidos en su dimensión espacial, esto es, en cuanto a las diferencias que presentan en distintos ámbitos

${ }^{33}$ Aunque esta tendencia se ha reducido en las últimas elecciones, éste sería un factor importante a la hora de tratar de explicar lo reducido de la victoria electoral del Partido Popular en las elecciones de 1996 respecto de los resultados alcanzados por el Partido Socialista, pese a darse unas condiciones objetivamente favorables para que la competitividad hubiera sido menor. 
geográficos - regiones, Comunidades Autónomas o circunscripciones- de forma simultánea. De hecho, ésta es una dimensión que distingue al sistema de partidos español entre sus homólogos europeos, al presentar peculiaridades regionales que obligan a hablar de variados subsistemas de partidos dentro del modelo general estatal. Se trata de arenas electorales diferenciadas, en las que la presencia de partidos políticos de ámbito no estatal compitiendo con distinto grado de éxito con los estatales modifica enormemente las pautas de competencia electoral y de interacción partidista. Esta peculiaridad del sistema de partidos español llevó hace años a Vallès (1991: 33 ss.) a acuñar el término de las «Españas electorales» para referirse a esas distintas y particulares arenas de competición electoral y partidista no estatal.

Para estudiar esta dimensión de los sistemas de partidos proponemos cuatro índices: el índice del voto regionalista, que informa acerca de la cantidad total de voto que reciben en el área geográfica que estudiamos los partidos de ámbito no estatal (PANE). El del voto regionalista diferenciado, que mide la relevancia o el peso que el voto específicamente regionalista o nacionalista tiene en el comportamiento electoral agregado en un ámbito electoral dado, en comparación con el que ese voto regionalista o nacionalista registra en un ámbito electoral superior o general. El del voto regional diferenciado (Lee, 1988), que pone de manifiesto la medida en la que la distribución agregada del voto a los partidos (todos) en un determinado ámbito (Comunidad Autónoma o provincia/circunscripción electoral) se diferencia de la distribución agregada del voto registrada en un ámbito superior (el Estado o una Comunidad Autónoma, respectivamente $)^{34}$. De forma complementaria, el programa INDELEC permite calcular el indice de escisión del voto, de Arian y Weiss (1969), que pondrá de manifiesto la medida en la que los ciudadanos de un ámbito dado votan por partidos distintos en diferentes tipos de elecciones.

Los cuatro índices miden la misma realidad, aunque poniendo el acento en distintas facetas. Dadas las limitaciones de espacio a las que este artículo se ve sometido, tampoco podemos ofrecer aquí un ejemplo de la aplicación del programa que ofrecemos para estas utilidades. En la monografía anunciada serán estudiados y aplicados tanto en el nivel de las Comunidades Autónomas como en el seno de cada una de ellas (entre sus distintas circunscripciones). Deberemos conformarnos aquí con anunciar su existencia y potencial explicativo.

\section{CONCLUSIONES}

En estas líneas hemos intentado presentar un instrumento que, consideramos, facilitará enormemente el trabajo de los estudiosos de los sistemas electorales y de partidos, al tiempo que le conferirá un máximo grado de frabilidad

${ }_{34}$ El índice fue inicialmente propuesto por Lee (1988) y aplicado, posteriormente, por Hearl, Budge y Pearson (1996: 169 ss.). 
(al quedar excluida la posibilidad de errores de cálculo). Se trata de un programa computacional que permite calcular rápida y sencillamente la desproporcionalidad electoral que se registra en un ámbito dado (distrito, Comunidad Autónoma o Estado), así como los valores de las principales dimensiones del sistema de partidos: la fragmentación, la competitividad, la polarización, la volatilidad y la dimensión regional.

El programa estará a disposición de los usuarios en la página web del CIS (http://www.cis.es) a principios del mes de octubre, después de su presentación oficial en el IV Congreso Nacional de la Asociación Española de Ciencia Política y de la Administración. En este mismo Congreso se presentará una monografía titulada Análisis electoral, publicada igualmente por el CIS, en la que, por un lado, se analizan detenidamente cada uno de los índices propuestos y sus ventajas e inconveniente; por otro, se aplican los distintos índices a todas las elecciones celebradas hasta la fecha en España, en un intento por confeccionar un atlas electoral analítico en distintas dimensiones y diferentes niveles (Estado, Comunidades Autónomas, circunscripciones y principales ciudades).

En estas páginas, a modo de ejemplo, hemos aplicado algunas de las utilidades del programa propuesto a las elecciones al Congreso de los Diputados celebradas en España hasta la fecha. Se dibujan, así, las principales consecuencias del sistema electoral que rige ese tipo de elecciones (las altas tasas de desproporcionalidad que provoca y sus variaciones en función del tamaño de la circunscripción) y las características más relevantes de los sistemas de partidos que se han sucedido en el tiempo: sistemas poco fragmentados, con un pequeño número de partidos relevantes, con altas tasas de concentración electoral y parlamentaria, y con unos niveles de competitividad variables en función del distinto período en el que nos fijemos. Por último, un sistema de partidos relativamente poco cristalizado, al registrarse en su seno unas considerablemente altas tasas de volatilidad electoral total (aunque su disminución en los últimos procesos electorales podría indicar un mayor grado de consolidación); volatilidad total que se debe, en mayor medida, a transferencias de voto entre partidos pertenecientes a un mismo bloque ideológico, lo que pone de relieve la relevancia que sigue teniendo en el sistema político estatal la dimensión izquierda-derecha a la hora de articularse el comportamiento político de sus ciudadanos. 


\section{REFERENCIAS BIBLIOGRÁFICAS}

ARIAN y WeISS (1969): «Split-ticket voting in Israel», Western Political Quarterly, 24 (375-389).

Baras, M., y Botella, J. (1996): El sistema electoral, Madrid, Tecnos.

Bartolini, S., y Mair, P. (1990): Identity, competition and electoral availability. The stabilisation of european electorates, 1885-1985, Cambridge, Cambridge University Press.

BlaIS, A., y CARTY, R. K. (1991): «The psychological impact of electoral laws: measuring Duverger's elusive factor», British Journal of Political Science, 21 (79-93).

Botella, J. (1998): «El sistema electoral español: fórmula electoral y umbrales de representación», en J. MONTABes (ed.), El sistema electoral a debate, Madrid, Centro de Investigaciones Sociológicas (91-100).

Castles, F. G., y Mair, P. (1984): «Left-right political scales: some "expert” judgments», European Journal of Political Research, 12 (73-88).

Cox, G. W., y SHUgarT, M. S. (1991): “Comment on Gallagher's "Proportionality, disproportionality and electoral system" ", Political Studies, 10 (348-352).

Duverger, M. (1972): Los partidos politicos, México, Fondo de Cultura Económica.

Gallagher, M. (1991): «Proportionality, disproportionality and electoral systems», Electoral Studies, vol. 10, 1 (33-51).

Gunther, R. (1989): «Leyes electorales, sistemas de partidos y élites: el caso español», Revista Española de Investigaciones Sociológicas, 47 (73-106).

Gunther, R.; SANI, G., y SHABAD, G. (1986): El sistema de partidos politicos en España: génesis y evolución, Madrid, Centro de Investigaciones Sociológicas.

Gunther, R., y Montero, J. R. (1994): «Los anclajes del partidismo: un análisis comparado del comportamiento electoral en cuatro democracias del sur de Europa», en P. DEL CASTILlO (ed.), Comportamiento politico y electoral, Madrid, Centro de Investigaciones Sociológicas (467-548).

Hazan, R. Y. (1997): Centre Parties: Polarization and Competition in European Parliamentary Democracies, London, Pinter.

Hearl, D. J.; Budge, I., y Pearson, B. (1996): «Distinctiveness of Regional Voting: A Comparative Analysis Across the European Comunity (1979-1993)», Electoral Studies, vol. 15, 2 (167-182).

Kesselman, M. (1966): «French Local Politics: A Statistical Examination of Grass Root Consensus», American Political Science Review, 60 (963-973).

Kuingemann, H. D. (1995): «Party positions and voter orientations», en H. D. Kuingemann y D. Fuchs (eds.), Citizens and the State, Oxford, Oxford University Press (183-205).

KRIESI, H. (1998): "The transformation of cleavege politics», European Journal of Political Research, 33 (165-185).

LeE, A. (1988): "The Persistence of Difference: Electoral Change in Cornwall», Political Studies Association Conference, Plymouth.

LIJPHART, A. (1986): «Degrees of proportionality of proportional representation formulas», en B. Grofman y A. Lijphart (eds.), Electoral laws and their political consequences, Nueva York, Agathon Press (170-179).

- (1994): Electoral systems and party systems. A study of twenty-seven democracies, 1945-1990, Oxford, Oxford University Press.

Lijphart, A., y Gibberd, R. W. (1977): «Thresholds and Payoffs in List System of Proportional Representation", European Journal of Political Research, vol. 5, 3 (219-244).

Loosemore, J., y Hanby, V. J. (1971): "The theoretical limits of maximum distortion: some analytical expressions for electoral systems», British Journal of Political Science, 1 (467-477).

Mackie, T. T., y Rose, R. (1982), The International Almanac of Electoral History, 2. ${ }^{\text {e }}$ ed., London, Macmillan.

- (1991): The International Almanac of Electoral History, 3. ${ }^{a}$ ed., London, Macmillan.

Molinar, J. (1991): "Counting the Number of Parties: An Alternative Index», American Political Science Review, vol. 85, 4 (1383-1391). 
Montero, J. R. (1994): «Sobre las preferencias electorales en España: fragmentación y polarización (1977-1993)», en P. DEL CASTILlo (ed.), Comportamiento politico y electoral, Madrid, Centro de Investigaciones Sociológicas (51-124).

- (1997): "Las elecciones legislativas», en R. Del ÁGuila (ed.), Manual de Ciencia Politica, Madrid, Trotta (391-428).

- (1998): «El debate sobre el sistema electoral: rendimientos, criterios y propuestas de reforma», en J. Montabes (ed.), El sistema electoral a debate, Madrid, Centro de Investigaciones Sociológicas (37-70).

Montero, J. R., y VAllès, J. M. (1992): «El debate sobre la reforma electoral: ¿para qué las reformas?», Claves de Razón Práctica, 22 (2-11).

Montero, J. R., y GunTHer, R. (1994): «Sistemas “cerrados” y listas “abiertas”: sobre algunas propuestas de reforma del sistema electoral en España”, en J. R. MONTERo et al., La reforma del régimen electoral, Madrid, Centro de Estudios Constitucionales (13-87).

Montero, J. R., y OÑAte, P. (1995): «El sistema electoral», en R. Chueca y J. R. Montero (eds.), Elecciones autonómicas en Aragón, Madrid, Tecnos (65-84).

Montero, J. R.; Pallarès, F., y Oñate., P. (1995): «El subsistema de partidos», en R. Chueca y J. R. Montero (eds.), Elecciones autonómicas en Aragón, Madrid, Tecnos (193-236).

Morlino, L. (1995): «Political parties and democratic consolidation in Southern Europe», en R. Gunther, P. N. Diamandouros y H. J. Puhle (eds.), The politics of democratic consolidation. Southern Europe in comparative perspective, London, Johns Hopkins University Press (261-283).

Oppenhuis, E. (1995): Voting behavior in Europe, Amsterdam, Het Spinhuis.

PALlarès, F. (1981): «La distorsión de la desproporcionalidad en el sistema electoral español. Análisis comparado e hipótesis alternativas», Revista de Estudios Políticos, 23 (233-267).

Pedersen, M. N. (1979): «The dynamics of West European party systems: Changing patterns of electoral volatility", European Journal of Political Research, 7 (1-26).

- (1983): «Changing patterns of electoral volatility in European party systems, 1948-1977: Explorations and explanations», en H. DAALDER y P. MAIR (eds.), Western European party systems. Continuity and change, Londres, Sage (29-66).

Pennini, A. (1998): «Disproportionality Indexes and Robusteness of Proportional Allocation Methods", Electoral Studies, vol. 17, 1 (3-19).

RAE, D. W. (1971): The political consequences of electoral laws, New Haven, Yale University Press.

- (1993): "Análisis del sistema electoral español en el marco de la representación proporcional», en D. W. RAE y V. Ramírez, Quince años de experiencia. El sistema electoral español, Madrid, McGraw-Hill.

RAMíreZ, V., et al. (1998): «Proporcionalidad y bonificación al partido vencedor», en J. MONTABES (ed.), El sistema electoral a debate, Madrid, Centro de Investigaciones Sociológicas (101-125).

SANI, G., y SARTORI, G. (1983): «Polarization, fragmentation and competition in Western democracies", en H. DAAlder y P. MaIr (eds.), Western European party systems. Continuity and change, Londres, Sage (307-340).

SAni, G., y Montero, J. R. (1986), "El espectro político: izquierda, derecha y centro», en J. J. LinZ y J. R. MONTERo (eds.), Crisis y cambio. Electores y partidos en la España de los años 80, Madrid, Centro de Estudios Constitucionales (155-200).

SANI, G., y SHABAD, G. (1986): «¿Adversarios o competidores?: La polarización del electorado», en J. J. Linz y J. R. Montero (eds.), Crisis y cambio. Electores y partidos en la España de los años 80, Madrid, Centro de Estudios Constitucionales (587-624).

SARTORI, G. (1987): Partidos y sistemas de partidos, Madrid, Alianza.

TAAGEPERA, R. (1997): "The effective number of parties for incomplete data», Electoral Studies, $16(145-151)$.

TAagepera, R., y LAaKso, M. (1980): «Proportional profiles of West European electoral systems", European Journal of Political Research, 8 (423-446).

TaAgepera, R., y Shugart, M. S. (1989): Seats and Votes. The effects and determinants of electoral systems, New Haven, Yale University Press. 
TukeY, J. W. (1977): Exploratory Data Analysis, Mass., Addison Wesley.

VALLÈS, J. M. (1991): «Entre la irregularidad y la indeterminación: balance sobre el comportamiento electoral en España (1977-1989)», en J. Vidal Beneyto (ed.), España a debate. La política, Madrid, Tecnos.

- (1998): «El número de representantes y la dimensión de las circunscripciones», en J. MONTABES (ed.), El sistema electoral a debate, Madrid, Centro de Investigaciones Sociológicas (77-90).

VAlLÈs, J. M., y BosCH, A. (1997): Sistemas electorales y gobierno representativo, Barcelona, Ariel.

VAN DER EIJK, C.; Franklin, M., y MARSH, M. (1996): «What voters teach us about Europe-wide elections: what Europe-wide elections teach us about voters», Electoral Studies, 15 (149-166).

Wert, J. I. (1996): «Las elecciones legislativas del 3-M. Paisaje para después de una batalla», Claves de Razón Práctica, 61 (36-44).

Wildgen, J. (1971): «The Measurement of Hyperfractionalization», Comparative Political Studies, 4 (233-243).

\begin{abstract}
This paper presents a computer programme which will be available to users on the CIS's web-page (http://www.cis.es) on October 1st. The INDELEC programme makes it possible to calculate the most important rates of disproportionality in election systems, and the most widespread with a view to gauging the dimensions of the party systems: fragmentation, number of parties, concentration, competitiveness, polarization, volatility, regional vote and dual vote. In this paper, and by way of example, the INDELEC programme is applied to the aggregate results of the parliamentary elections which have been held in Spain since 1977. In a monograph entitled Analisis electoral which will be published in September, each indicator will be examined in depth and note taken of its advantages and drawbacks, and they are applied to the different types of elections which have taken place in Spain since the reinstatement of democracy and at a different level of aggregation and disaggregation. Thus, the authors draw a map with the main characteristics of the various party systems and sub-systems (state and regional) which have existed in Spain since 1977.
\end{abstract}

